\title{
A Search for Short-Term RV Variations in Early-Type Binaries
}

\author{
H. Lehmann \\ Thüringer Landessternwarte Tautenburg, Germany \\ G. Hildebrandt, G. Scholz \\ Astrophysikalisches Institut Potsdam, Germany
}

\begin{abstract}
We developed a procedure to disentangle the different radial velocity variations due to orbital motion, rotation, and pulsation based on an iterative mutual subtraction of orbital and short-term contributions. The method was successfully tested on a data set of over 2200 spectra of the eclipsing $\beta$ Cep star EN Lac spanning a time interval of about 90 years. We further applied the procedure to early type binaries. For the A1 III star 21 Her we found short-term variations which indicate the presence of rotationally split nonradial pulsation (NRP) modes.
\end{abstract}

\section{Introduction}

The investigation of stellar pulsations in spectroscopic binaries requires a very careful discrimination between velocity variations caused by orbital motion, by rotation, and by pulsation. For stars with large pulsation amplitudes, e.g. the $\beta$ Cep stars, an exact spectroscopic orbit can only be determined if the pulsational contributions to the observed radial velocity (RV) variations are precisely known. Low amplitude pulsations can only be detected after a correct subtraction of the orbital RV variations. This situation renders even more severe if we consider multiple frequency spectra of pulsating stars.

\section{The applied method}

Our method is based on an iterative mutual subtraction of orbital and short-term RV contributions. Three different computer programs are used. The program computing the orbital solution is based on the method of iterative differential corrections of the orbital elements and the orbital period. It considers also contributions due to apsidal motion and/or by a variable period length. The program handles weighted data and determines the different instrumental RV zero-points of different data groups. The second program is used to find additional periods and computes the periodogram of the residuals after subtracting the orbital solution and all previously found additional RV variations. The ordinate of the resulting periodogram gives a measure of the reduction in the sum of squares obtained by the applied sinewave fit (Lehmann et al., 1999). The third 
program optimizes the frequencies, amplitudes, and phases of all contributions found at every step of successive pre-whitening simultaneously. It considers also a given number of harmonics of each frequency. Amplitude modulation is taken into account by searching for possible contributions of sidelobes of the considered frequencies.

\section{Results}

The method was successfully tested on a data set of over 2200 spectra of the eclipsing $\beta$ Cep star EN Lac spanning a time interval of about 90 years which includes very inhomogeneous data taken by different authors with different instruments and detectors. From the disentangling of orbital and short-term variations we got a much more precise orbital solution and more accurate values of the three known main pulsational modes having periods of $0.16916703,0 \mathrm{~d} 17085554$, and 0 d 18173256. Moreover, we found for all three variations time scales of amplitude modulation having values of $74 \mathrm{y}, 331 \mathrm{~d}$, and $674 \mathrm{~d}$, respectively (Lehmann et al., 2001a).

We further applied the procedure to early type binaries with orbits of different excentricity to search for a possible connection between orbital motion and the excitation of pulsations. For $21 \mathrm{Her}$ (A1 III, orbital period of 5.02) we found short-term RV variations possibly caused by pulsation. The observed changes of the orbital residuals can be described by four variations with periods of $58 \mathrm{~d}$, $1.48,0 \mathrm{~d} 21060$, and 0.22382 . The first period could be due to a third body in the 21 Her system having the mass of a brown dwarf and the second could be related to half of the rotational period of $21 \mathrm{Her}$. The frequency difference of the two short-term periods corresponds to a period of 3.6 and could be related to the rotational period of $21 \mathrm{Her}$ as expected from the measured $v \sin i$ of $60 \mathrm{~km} \mathrm{~s}^{-1}$. Thus the observed short-term oscillations can be interpreted as rotationally split NRP modes. In the case of $21 \mathrm{Her}$ a very careful disentangling of orbital and additional RV variations was necessary. Including our own and older observations of Harper (1934), the final solution allowed us to derive also a slight but significant increase of the orbital period with a rate of $0.34 \mathrm{sec}^{-1}$ (Lehmann et al., 2001c).

For the other two program stars investigated so far, $\gamma \mathrm{Gem}$ (A0 IV) and HD 169981 (A2 III), we did not find any short-term variations; however, we found some remarkable results from the new orbital solutions. By combining our RVs with own and Hipparcos photometry we could show that HD 169981 is very probably an eclipsing binary consisting of a A2 III primary and a late type secondary of K3 V (Lehmann et al., 2001b).

\section{References}

Harper, W.E. 1934, Publ. Dom. Astrophys. Obs. 6, 235

Lehmann, H., Scholz, G., Hildebrandt, G., \& Panov, K. 1999, A\&A, 351, 267

Lehmann, H., Harmanec, P., Aerts, C., et al. 2001a, A\&A, 367, 236

Lehmann, H., Hildebrandt, G., Panov, K.P., \& Scholz, G. 2001b, A\&A, 373, 960

Lehmann, H., Andrievsky, S.M., Egorova, I., et al. 2001c, A\&A, in press 